\title{
Endothermic and Exothermic Energy Transfer Made Equally Efficient for Triplet-Triplet Annihilation Upconversion
}

Jussi Isokuortti ${ }^{1}$, Srinivasa Rao Allu², Alexander Efimov ${ }^{1}$, Elina Vuorimaa-Laukkanen ${ }^{1}$, Nikolai V. Tkachenkol, Sergei A. Vinogradov ${ }^{2}$, Timo Laaksonen ${ }^{1,3}$ and Nikita A. Durandin ${ }^{1 *}$

${ }^{1}$ Faculty of Engineering and Natural Sciences, Tampere University, Korkeakoulunkatu 8, 33720 Tampere, Finland

${ }^{2}$ Department of Biochemistry and Biophysics, Perelman School of Medicine, and Department of Chemistry, School of Arts and Sciences, University of Pennsylvania, Philadelphia, PA 19104, USA

${ }^{3}$ Division of Pharmaceutical Biosciences, Faculty of Pharmacy, University of Helsinki, Viikinkaari 5 E, 00014, Helsinki, Finland

\section{Contents}

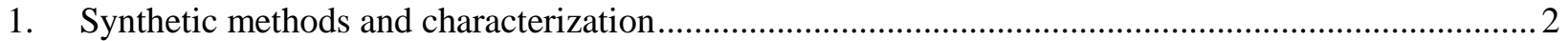

2. Viscosity measurements of the poly(ethylene glycols) ................................................................. 2

3. Photophysical measurements and kinetic rate modelling .............................................................. 2

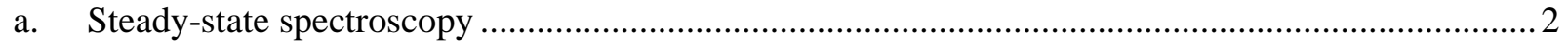

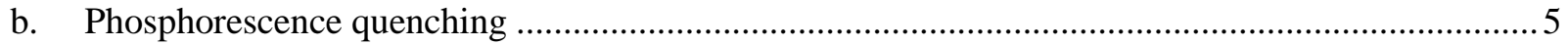

c. Dependence of upconversion fluorescence intensity on annihilator concentration and excitation

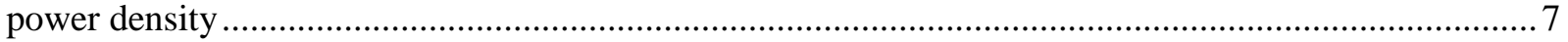

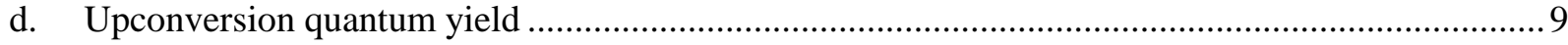

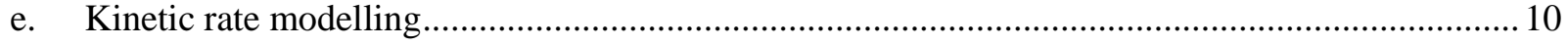

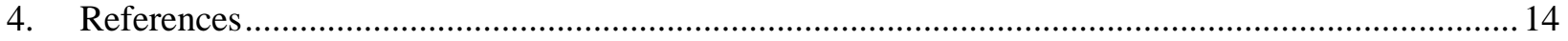




\section{Synthetic methods and characterization}

\section{Palladium(II) and zinc(II) N-\{2,6-di[(3'(methoxycarbonyl)propyloxy)phenyl]\} phthalimido- porphyrin (PdTAPIP and ZnTAPIP)}

Synthesis and characterization have been reported elsewhere. ${ }^{1}$

\section{9-(4-phenylethynyl)-10-phenylanthracene (PEAP)}

Synthesis and characterization have been reported elsewhere. ${ }^{2}$

\section{Viscosity measurements of the poly(ethylene glycols)}

The dynamic viscosities of both PEG200 (at $23{ }^{\circ} \mathrm{C}$ ) and PEG300 (at $-5{ }^{\circ} \mathrm{C}$ ) were measured by using an Anton Paar MCR 301 rotational rheometer.

\section{Photophysical measurements and kinetic rate modelling}

\section{a. Steady-state spectroscopy}

All the measurements were carried out using freshly prepared dichloromethane (DCM), PEG200 or PEG300 solutions. UV-Vis absorption spectra were recorded using a Shimadzu UV-2501PC (Shimadzu, Japan) spectrophotometer with a 1-cm quartz cell. Steady-state fluorescence and phosphorescence spectra were recorded with an FLS-1000 (Edinburgh Instruments, UK) spectrofluorometer equipped with double excitation and emission monochromators.

Absorption spectra of PEAP in both DCM and PEG200 are shown in Figures S1 and S2, respectively. Absorption spectra of PdTAPIP and ZnTAPIP in DCM are shown in Figure S3. Extinction coefficients of all molecules are shown in Table S1.

Fluorescence spectra of PEAP in DCM and PEG200 is shown in Figure S4. Luminescence spectra of PdTAPIP and ZnTAPIP in PEG200 are shown in figures S5 and S6, respectively. For phosphorescence measurements the samples were deoxygenated by purging 1 hour with nitrogen and adding $50 \mathrm{mM}$ of oleic acid. 


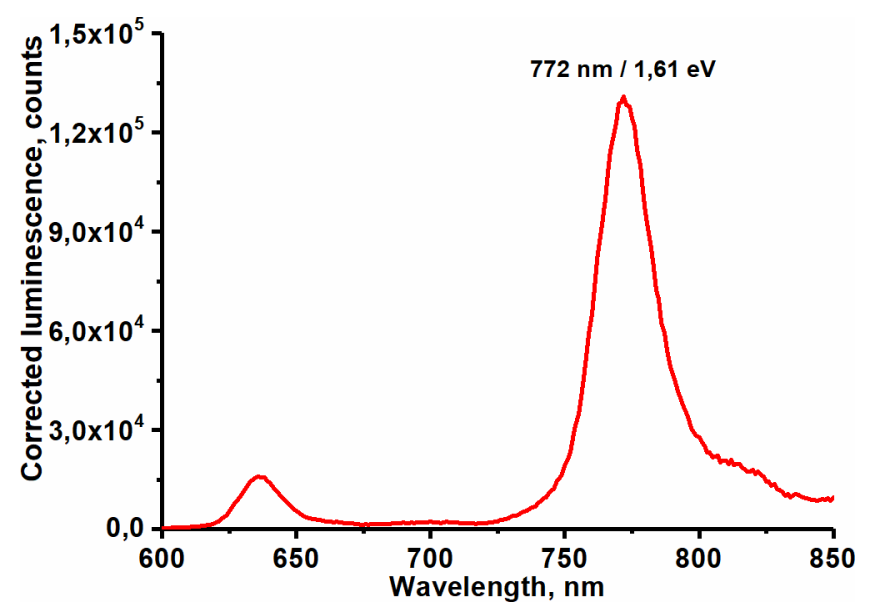

Figure S1. Luminescence spectra of PdTAPIP in deoxygenated PEG200. The small peak at $635 \mathrm{~nm}$ is delayed fluorescence of PdTAPIP.

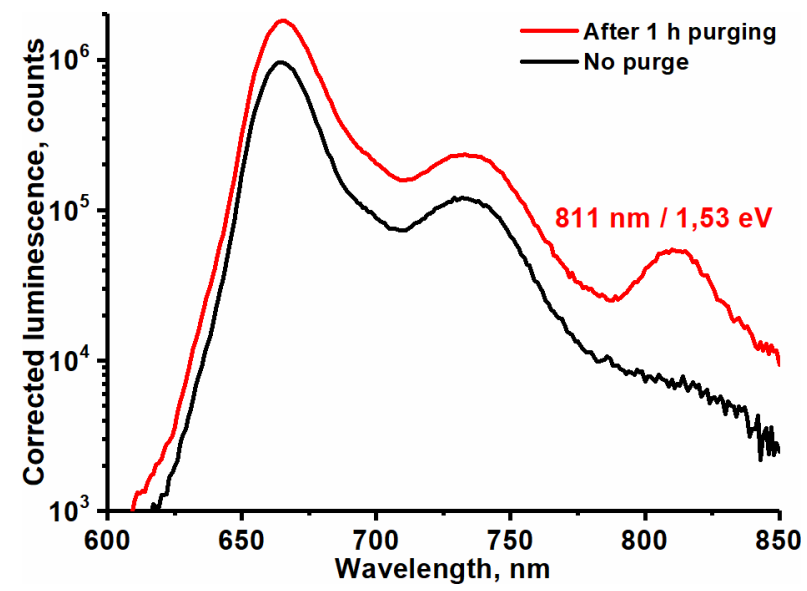

Figure S2. Luminescence spectra of ZnTAPIP in regular and deoxygenated PEG200. The spectrum measured in regular PEG200 shows ZnTAPIP fluorescence and in deoxygenated PEG200 the phosphorescence is distinct.

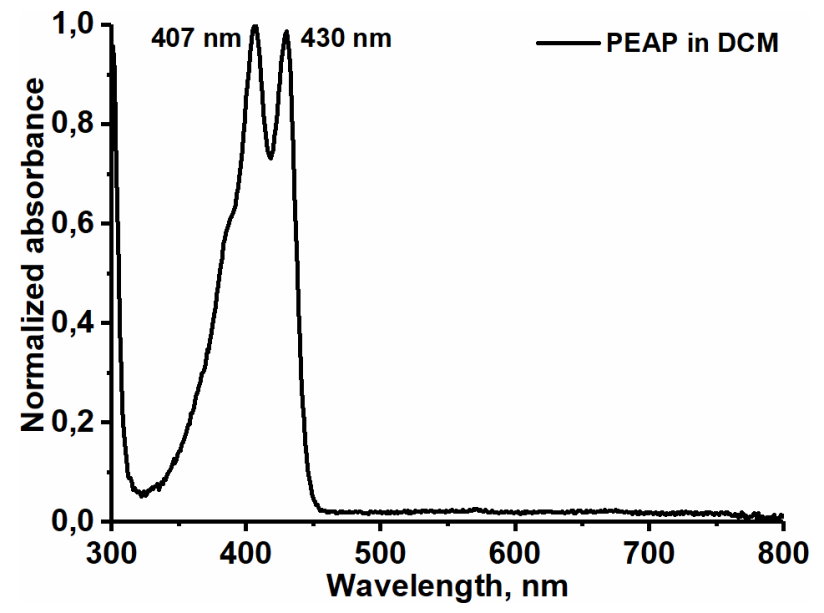

Figure S3. Absorption spectrum of PEAP in DCM. 


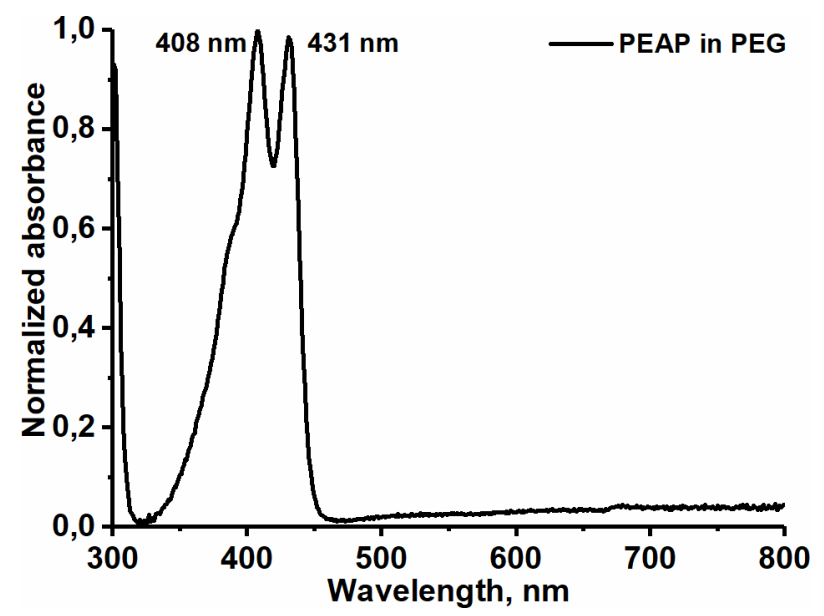

Figure S4. Absorption spectrum of PEAP in PEG200.

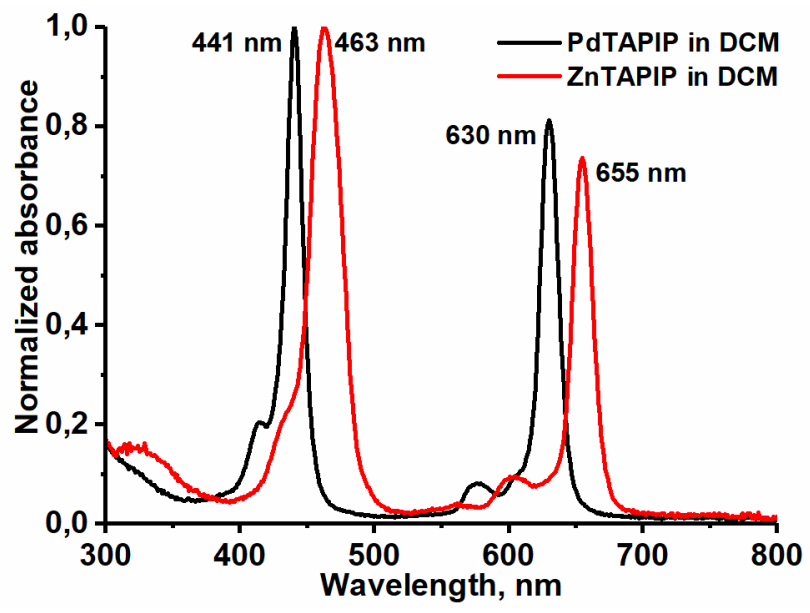

Figure S5. Absorption spectra of PdTAPIP and ZnTAPIP in DCM.

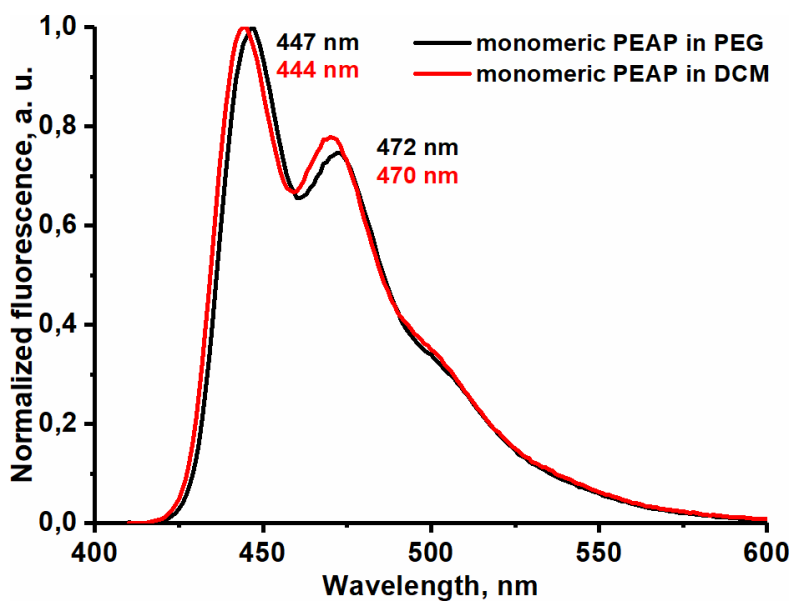

Figure S6. Fluorescence spectra of PEAP in DCM and PEG200. 
Table S1. Molar exticntion coefficients of PEAP, PdTAPIP and ZnTAPIP.

\begin{tabular}{c|cc}
\hline & $\boldsymbol{\epsilon}$ in DCM, $\mathbf{M}^{\mathbf{- 1}} \mathbf{c m}^{\mathbf{- 1}}$ & $\boldsymbol{\epsilon}$ in PEG200, $\mathbf{M}^{\mathbf{- 1}} \mathbf{c m}^{\mathbf{- 1}}$ \\
\hline PdTAPIP, 441 nm / 444 nm & 342900 & 342900 \\
PdTAPIP, 630 nm / 630 nm & 263500 & 263500 \\
ZnTAPIP, 463 nm / 476 nm & 271900 & 356300 \\
ZnTAPIP, 655 nm / 659 nm & 191200 & 198500 \\
PEAP, 407 nm / 408 nm & 21000 & 21000 \\
PEAP, 430 nm / 431 nm & 20600 & 20700 \\
\hline
\end{tabular}

Fluorescence quantum yield of ZnTAPIP in PEG200 was measured using FLS1000 and an integrating sphere (Edinburg Instruments, UK). The sample had an optical density of 0.1 at the excitation wavelength of $659 \mathrm{~nm}$.

\section{b. Phosphorescence quenching}

Phosphorescence decays of PdTAPIP and ZnTAPIP in PEG200 and PEG300 were measured in presence of PEAP as quencher and shown in Figures S7-S10. Decays were recorded with FLS1000 using $\mu$ F flash lamp. Samples were prepared in a $1 \mathrm{~cm} 2$ special optical glass cuvettes with screw cap and septum (Starna Scientific Ltd., UK). Deoxygenation was performed using nitrogen purging for ca. 1 hour and adding 50 $\mathrm{mM}$ of oleic acid. The concentrations of PdTAPIP and ZnTAPIP were $0.4 \mu \mathrm{M}$ and $0.5 \mu \mathrm{M}$ to yield samples with optical density of 0.1 at excitation wavelengths $(630 \mathrm{~nm}$ and $659 \mathrm{~nm})$, respectively. The decays were monitored at the phosphorescence maxima of PdTAPIP $(772 \mathrm{~nm})$ and ZnTAPIP $(811 \mathrm{~nm})$. The phosphorescence detection of ZnTAPIP was gated $(10 \mu \mathrm{s})$ to exclude the fluorescence signal from the decays. Monoexponential tail fitting of the decays was performed with the Fluoracle program (Edinburgh Instruments, UK). With PEG300 samples, the sample holder inside FLS1000 was cooled with a Huber Ministat 125 (Germany) cooling circulator.

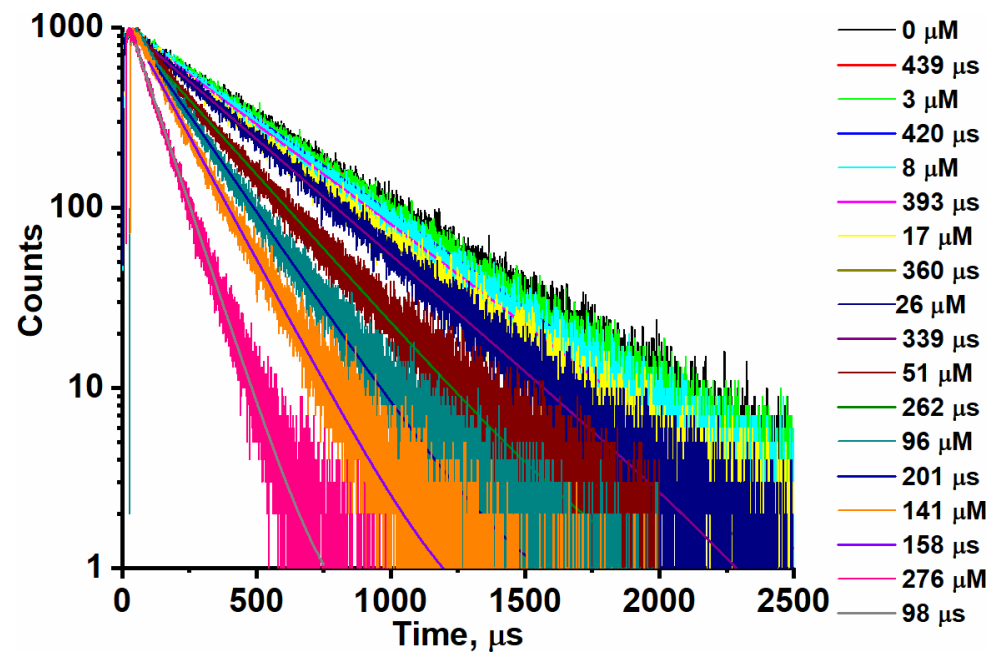

Figure S7. Phosphorescence decays of PdTAPIP in PEG200. Excitation at $630 \mathrm{~nm}$. 


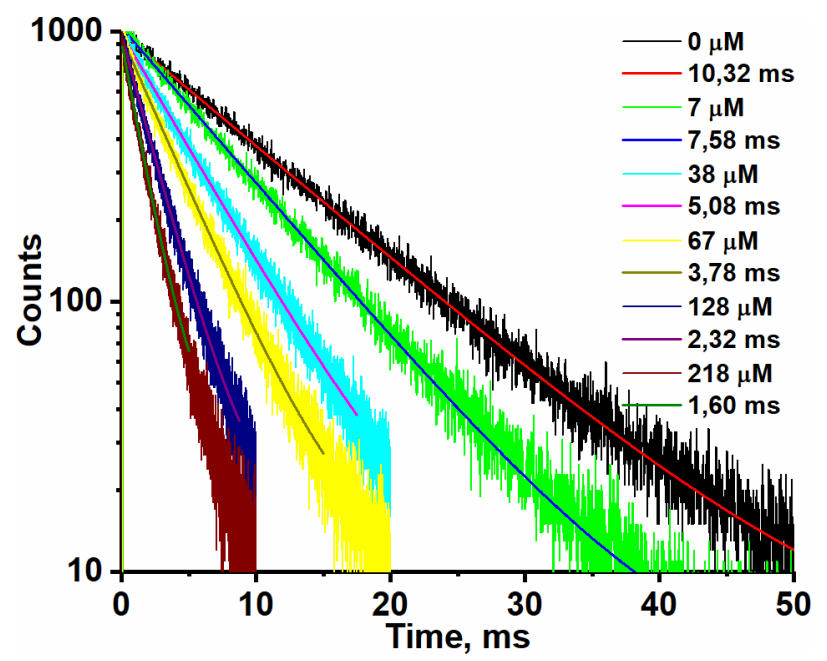

Figure S8. Phosphorescence decays of ZnTAPIP in PEG200. Excitation at $659 \mathrm{~nm}$.

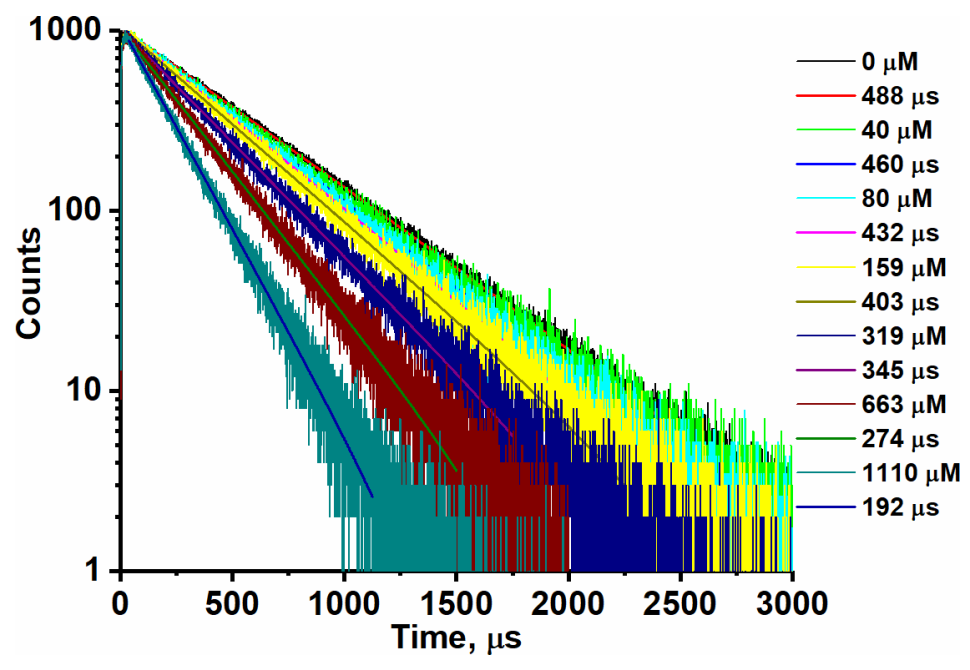

Figure S9. Phosphorescence decays of PdTAPIP in PEG300. Excitation at $630 \mathrm{~nm}$.

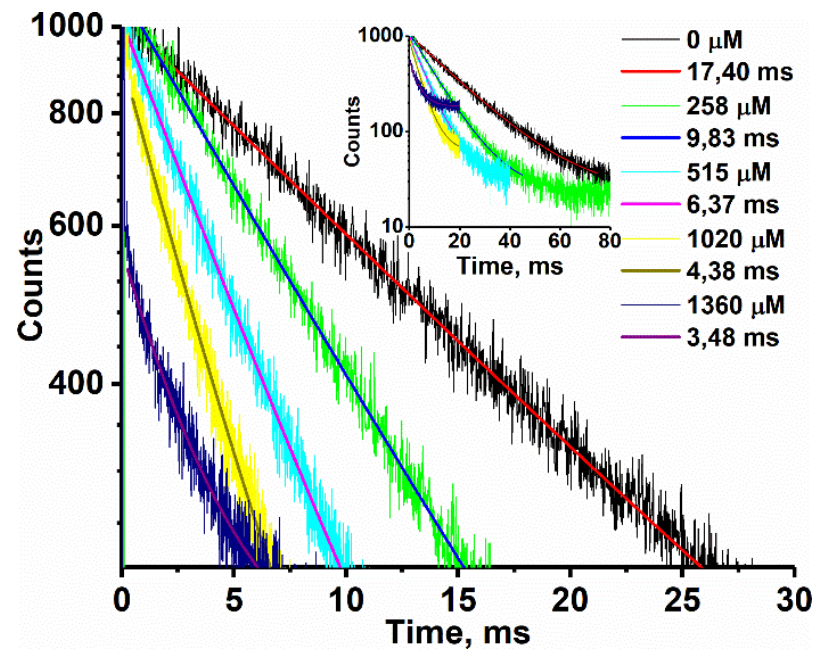

Figure S10. Phosphorescence decays of ZnTAPIP in PEG300. Excitation at $659 \mathrm{~nm}$. 


\section{c. Dependence of upconversion fluorescence intensity on annihilator concentration and excitation power density}

Samples with PdTAPIP were excited with a Helium-Neon laser (HNL210LB, $21 \mathrm{~mW}$, polarized, Thorlabs inc. USA) and ZnTAPIP samples were excited with a modulated laser diode (06-MLD $660 \mathrm{~nm}, 100 \mathrm{~mW}$, Cobolt, Sweden). To measure the laser power at the sample, we used a PM100D Digital Optical Power and Energy Meter (Thorlabs inc. USA) and to calculate the laser power densities the laser beam profiles and dimensions were measured with a LBP2-HR-VIS2 Laser Beam Profiler (Newport) and determined with LBP2 Software (Newport) and its Auto-aperture feature.

The dependence of upconversion emission intensity on annihilator concentration were measured using above mentioned lasers and FLS1000 as a sample chamber and detector. Laser beams were focused to a spot size that yielded $6 \mathrm{~W} / \mathrm{cm}^{2}$ and power was attenuated with neutral density filters (Edmund Optics, USA). Sensitizer concentrations $(4.2 \mu \mathrm{M}$ and $8.4 \mu \mathrm{M}$ of PdTAPIP and $5.1 \mu \mathrm{M}$ and $10.2 \mu \mathrm{M}$ of ZnTAPIP in PEG200 and PEG300, respectively) and excitation power (and power density) were kept constant and well above the power density thresholds $\left(2 \mathrm{~W} / \mathrm{cm}^{2}\right.$ for PEG200 samples and $4 \mathrm{~W} / \mathrm{cm}^{2}$ for PEG300 samples).

The dependence of upconversion emission intensity on excitation power density were measured by altering the laser power (and thus power density) with neutral density filters. The resulting double logarithmic plots of UC fluorescence intensity versus excitation power density are shown figures S11-S14. To visualize the linear and quadratic dependence of upconversion fluorescence intensity to the excitation power density, fitted slopes of 1 and 2 are plotted in Figures S11-S14.

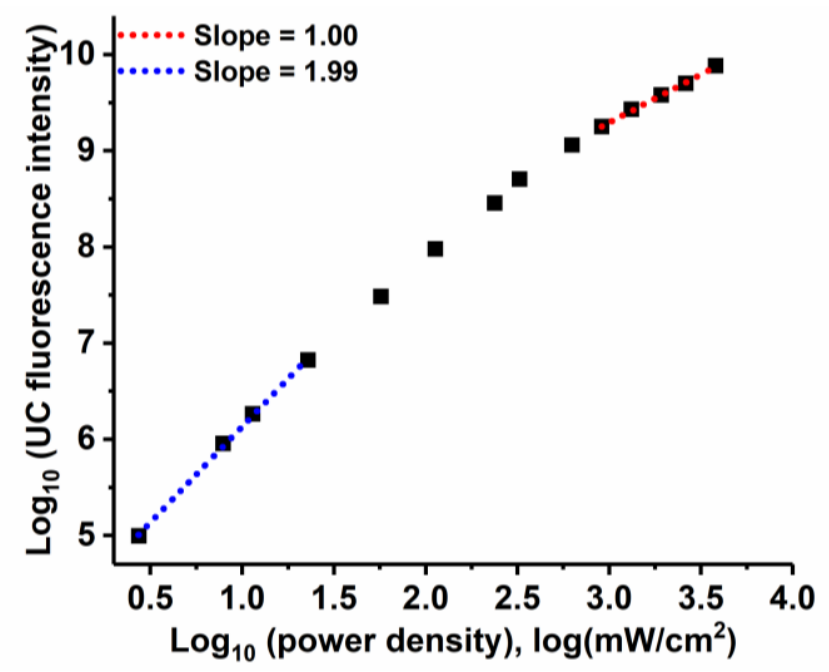

Figure S11. Upconversion fluorescence intensity of $4.2 \mu \mathrm{M}$ of PdTAPIP and 1.2 mM of PEAP in PEG200 under varied laser power density. 


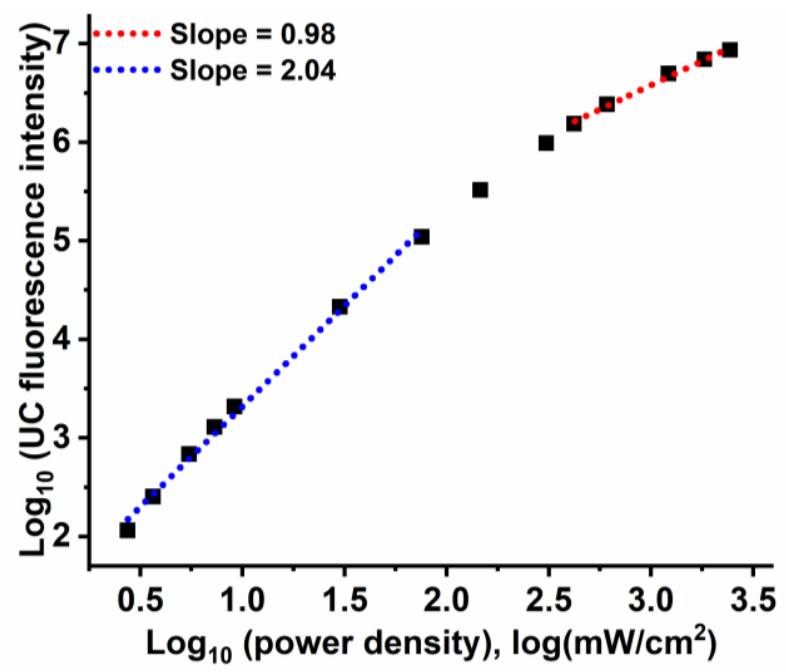

Figure S12. Upconversion fluorescence intensity of $5.1 \mu \mathrm{M}$ of ZnTAPIP and $5 \mathrm{mM}$ of PEAP in PEG200 under varied laser power density.

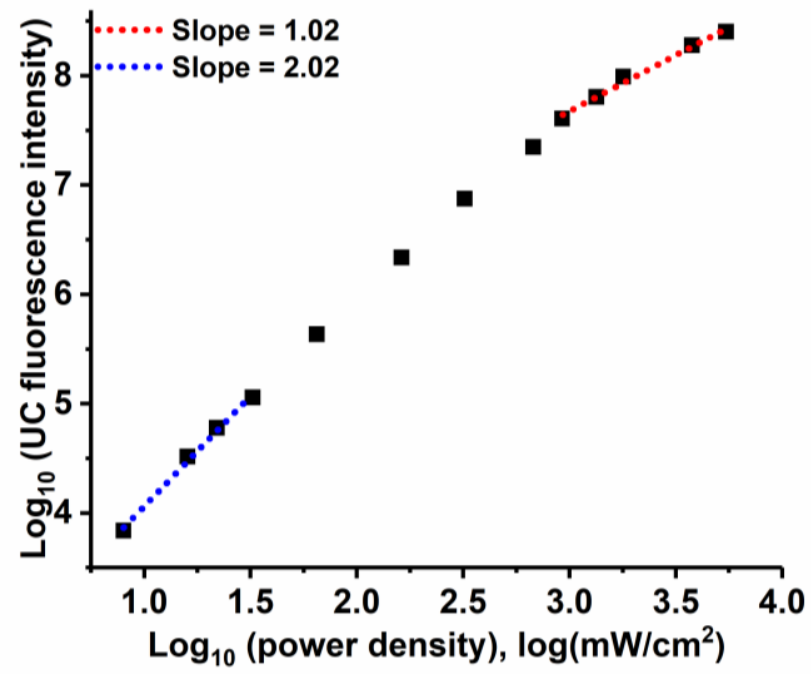

Figure S13. Upconversion fluorescence intensity of $8.4 \mu \mathrm{M}$ of PdTAPIP and $10 \mathrm{mM}$ of PEAP in PEG300 under varied laser power density. 


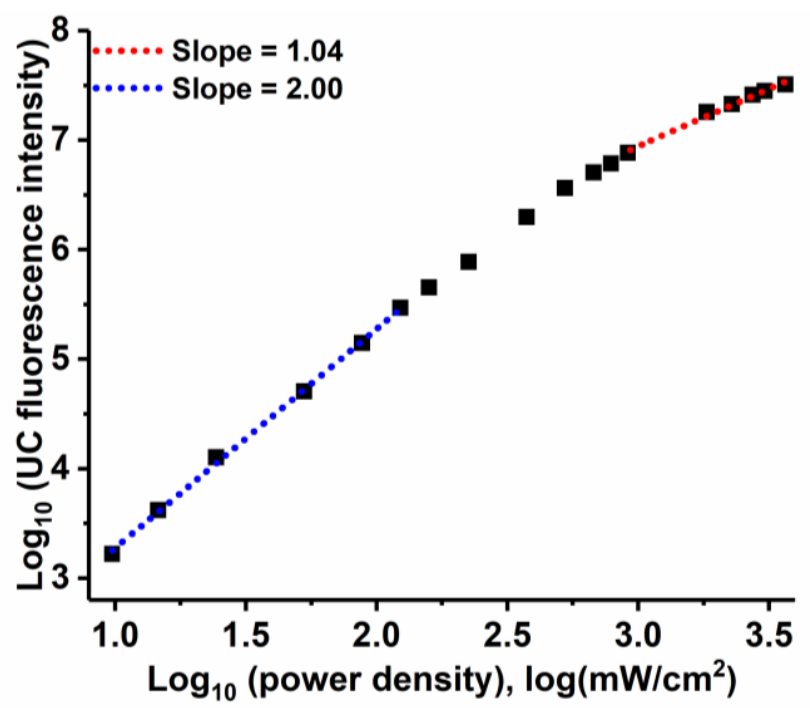

Figure S14. Upconversion fluorescence intensity of $10.2 \mu \mathrm{M}$ of ZnTAPIP and $20 \mathrm{mM}$ of PEAP in PEG300 under varied laser power density.

\section{d. Upconversion quantum yield}

Upconversion quantum yields were measured by using methylene blue $\left(\Phi_{\text {fluo }}=\Phi_{\text {std }}=0.04^{3}\right)$ as standard and comparing the integrated upconversion emission intensities to the integrated standard fluorescence intensities: ${ }^{4}$

$\Phi_{\mathrm{UC}}=2 \Phi_{\mathrm{std}}\left(\frac{\mathrm{A}_{\mathrm{std}}}{\mathrm{A}_{\mathrm{UC}}}\right)\left(\frac{\mathrm{I}_{\mathrm{UC}}}{\mathrm{I}_{\mathrm{std}}}\right)\left(\frac{\eta_{\mathrm{UC}}}{\eta_{\mathrm{std}}}\right)^{2}$

where $\mathrm{A}_{\text {std }}$ and $\mathrm{A}_{\mathrm{UC}}$ are the absorbances of the standard and the upconversion sample at the excitation wavelength, $I_{U C}$ and $I_{\text {std }}$ are the integrated emission intensities and $\eta_{U C}$ and $\eta_{\text {std }}$ are the refractive indices of the solvents used (PEG200 for upconversion samples and water for methylene blue). The factor 2 is added to Eq. S1 since TTAUC is a two-photon process. ${ }^{5}$ The concentration of the standard was kept low (absorbance of 0.1 at excitation wavelength) to ensure monomeric fluorescence and to minimize inner filter effect. The upconversion samples in PEG200 were prepared to have the same sensitizer and annihilator concentrations as the samples used for power density dependence measurements. Both the upconverted and standard fluorescence spectra were measured with a previously reported scheme $e^{6,7}$ using the above mentioned lasers and AvaSpec-2048 fiber-optic spectrometer (Avantes, Netherlands). The quantum yields of PEG300 samples were determined by comparing the upconversion fluorescence intensities of PEG200 and PEG300 samples recorded for the power density dependence measurements.

The upconverted fluorescence spectra of PdTAPIP and ZnTAPIP are shown in Figures S15 and S16 respectively. The upconverted fluorescence was attenuated by inner filter effect, so the recorded spectra were corrected by tail-fitting monomeric PEAP fluorescence (no excimer or aggregate fluorescence was observed with even as high as $20 \mathrm{mM}$ PEAP concentration) to the recorded spectra. The corrected spectra are also shown in Figures S15 and S16. 


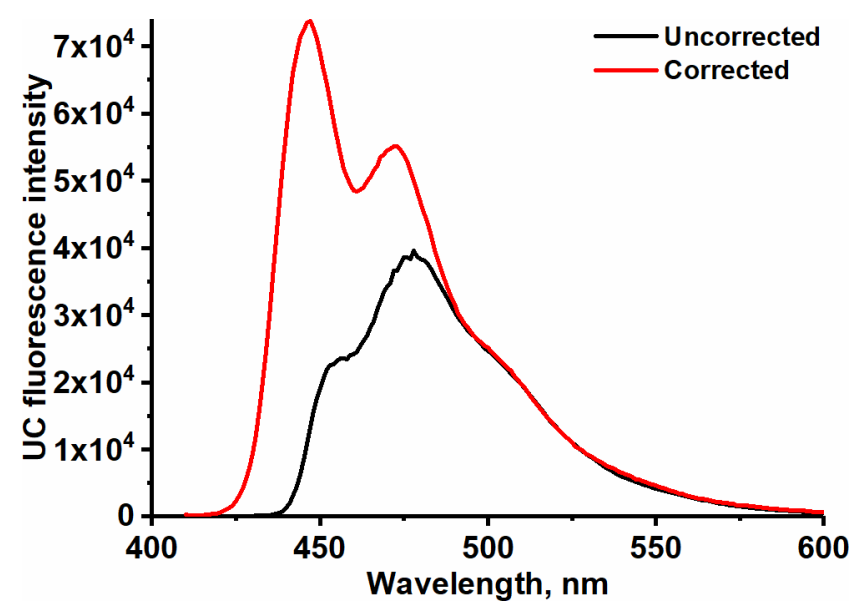

Figure S15. Uncorrected and corrected upconverted fluorescence of PEAP (1.2 $\mathrm{mM})$ sensitized by PdTAPIP $(4.2 \mu \mathrm{M})$ in deoxygenated PEG200 under $1500 \mathrm{~mW} / \mathrm{cm}^{2}$ excitation.

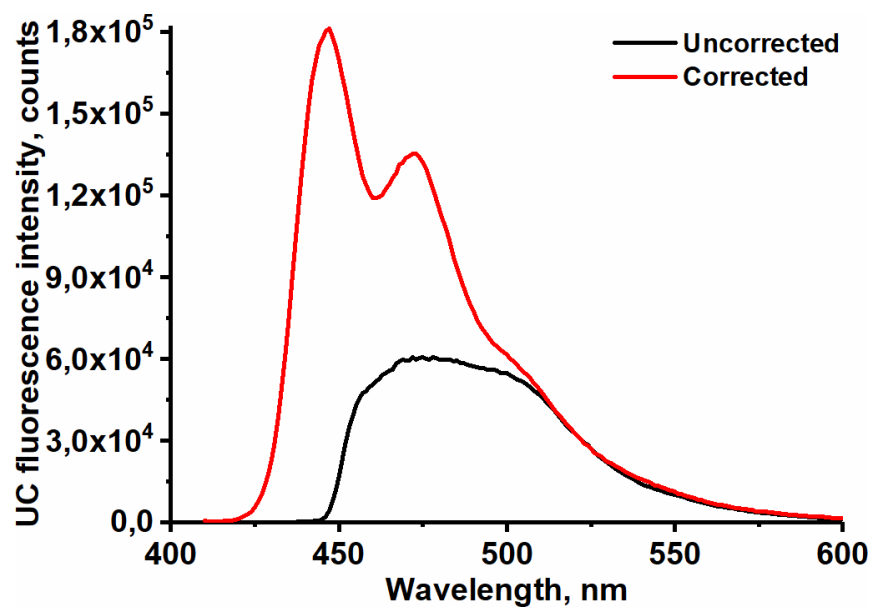

Figure S16. Uncorrected and corrected upconverted fluorescence of PEAP (5 mM) sensitized by ZnTAPIP $(5.1 \mu \mathrm{M})$ in deoxygenated PEG200 under $1500 \mathrm{~mW} / \mathrm{cm}^{2}$ excitation.

\section{e. Kinetic rate modelling}

Kinetic rate modelling was based on previously reported methods ${ }^{7}$. MATLAB R2018b software ("vpasolve" solver) has been used to solve equations S2-S4 numerically while varying sensitizer and annihilator concentrations.

$\frac{\mathrm{dS}_{3}^{*}}{\mathrm{dt}}=-\frac{\mathrm{dS}_{1}^{*}}{\mathrm{dt}}=\mathrm{k}_{\text {photon }}\left[\mathrm{S}_{0}\right] \Phi_{\mathrm{ISC}}-\mathrm{k}_{\mathrm{nr}}\left[\mathrm{S}_{3}^{*}\right]-\mathrm{k}_{\mathrm{FTET}}\left[\mathrm{S}_{3}^{*}\right]\left[\mathrm{A}_{0}\right]+\mathrm{k}_{\mathrm{RTET}}\left[\mathrm{A}_{3}^{*}\right]\left[\mathrm{S}_{0}\right]-\mathrm{k}_{\mathrm{TTA}}\left(2\left[\mathrm{~S}_{3}^{*}\right]+\left[\mathrm{S}_{3}^{*}\right]\left[\mathrm{A}_{3}^{*}\right]\right)=0$

$\frac{\mathrm{dA}_{3}^{*}}{\mathrm{dt}}=-\frac{\mathrm{dA}_{1}^{*}}{\mathrm{dt}}=-\mathrm{k}_{\mathrm{p}}\left[\mathrm{A}_{3}^{*}\right]+\mathrm{k}_{\mathrm{FTET}}\left[\mathrm{S}_{3}^{*}\right]\left[\mathrm{A}_{0}\right]-\mathrm{k}_{\mathrm{RTET}}\left[\mathrm{A}_{3}^{*}\right]\left[\mathrm{S}_{0}\right]-\mathrm{k}_{\mathrm{TTA}}\left(2\left[\mathrm{~A}_{3}^{*}\right]+\left[\mathrm{S}_{3}^{*}\right]\left[\mathrm{A}_{3}^{*}\right]\right)=0$

$\Phi_{\mathrm{UC}}=2 \frac{\mathrm{k}_{\mathrm{TTA}}\left[\mathrm{A}_{3}^{*}\right]^{2}}{\mathrm{k}_{\text {photon }}\left[\mathrm{S}_{0}\right]} \times \Phi_{\mathrm{fl}}^{\mathrm{A}}$ 
In the equations above, $\mathrm{S}$ and $\mathrm{A}$ are sensitizer and annihilator, subscripts 0,1 and 3 are ground, singlet and triplet state, respectively, $\mathrm{k}_{\mathrm{nr}}$ is the sensitizer's triplet decay rate constant, $\mathrm{k}_{\mathrm{p}}$ is the annihilator's triplet decay rate constant, $\Phi_{\mathrm{UC}}$ value of $32 \%$ was set to make calculations consistent between sets while keeping annihilator's fluorescence quantum yield also constant $64 \%$. $\mathrm{k}_{\mathrm{TTA}}$ was set as $50 \%$ of $\mathrm{k}_{\text {diff. }} \mathrm{k}_{\text {photon }}$ is the photon absorption rate (in $\mathrm{s}^{-1}$ ), which was converted to power density by using sensitizer's molar exctinction coefficient and excitation wavelength.

Annihilator's triplet decay rate constant used in the model was $\frac{1}{2.4 \times 10^{-3} \mathrm{~s}}$ in PEG200, the same value that Gray et al. have obtained in deoxygenated toluene for PEAP ${ }^{8}$. In PEG300 we used $\mathrm{k}_{\mathrm{p}}=\frac{1}{3.6 \times 10^{-3} \mathrm{~s}}$. This value was estimated based on how the triplet lifetimes of the sensitizer's increased in PEG300 compared to PEG200. The simulations based on the used value also gave good agreement to the experimental results.

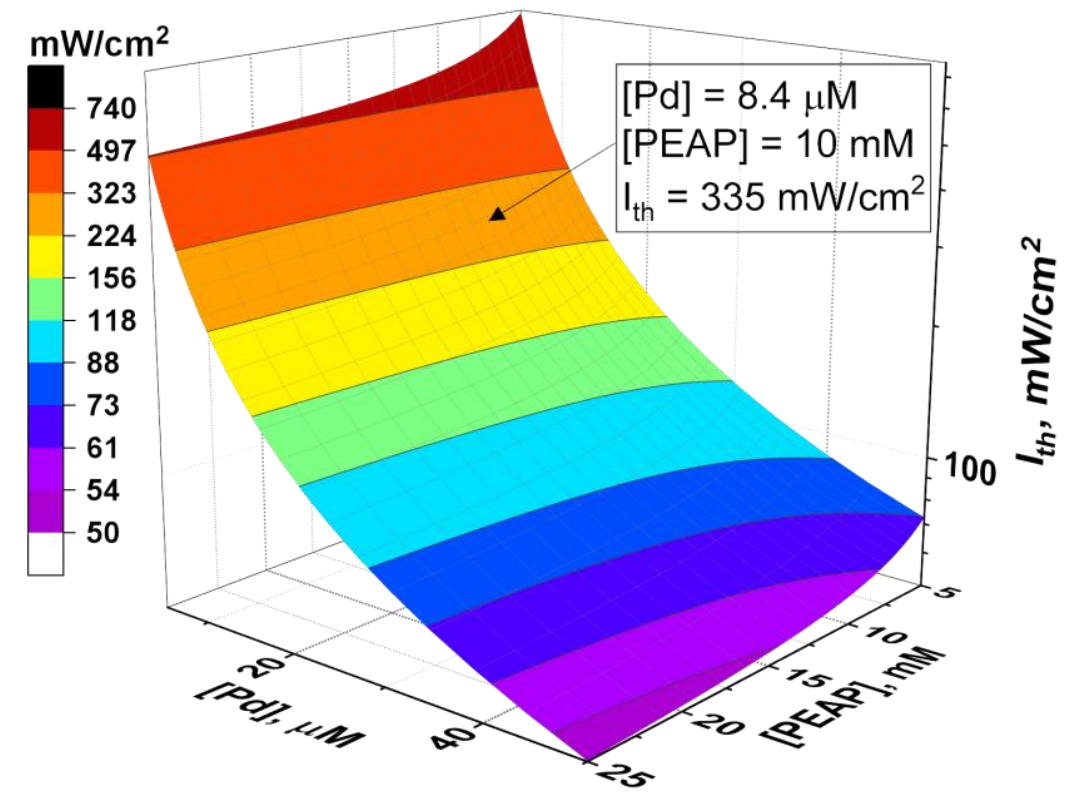

Figure S17. Kinetic rate model plot of PdTAPIP and PEAP in PEG300. 


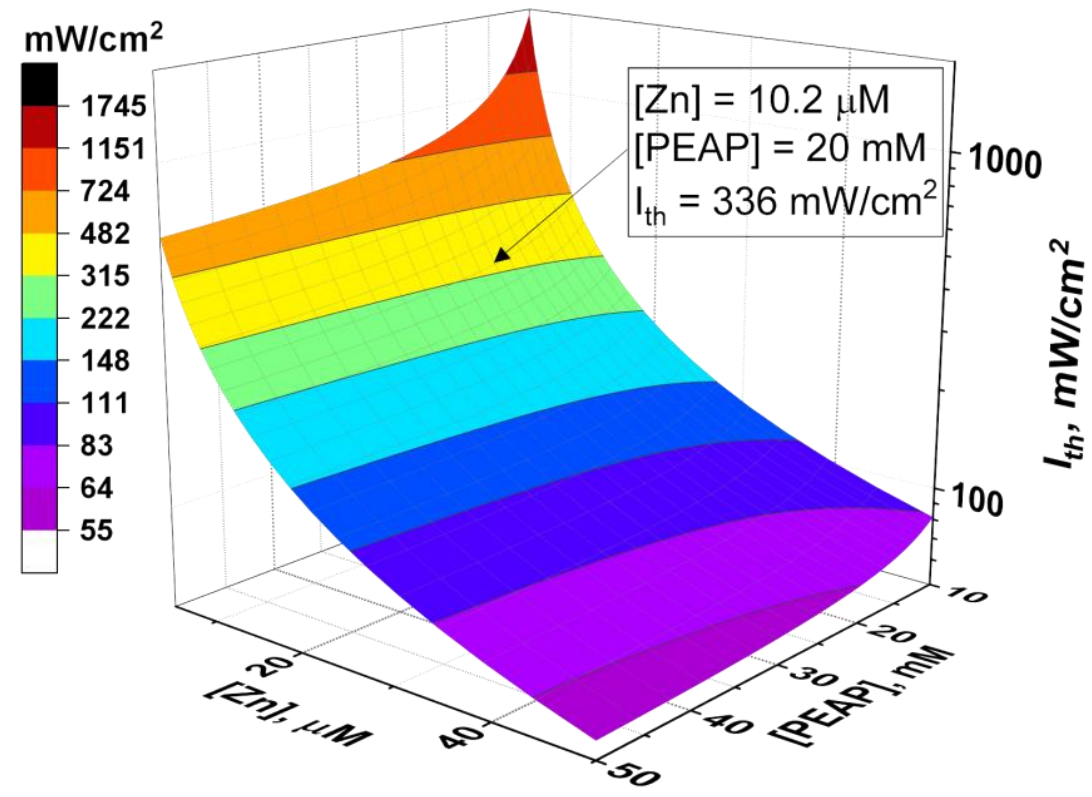

Figure S18. Kinetic rate model plot of ZnTAPIP and PEAP in PEG300.

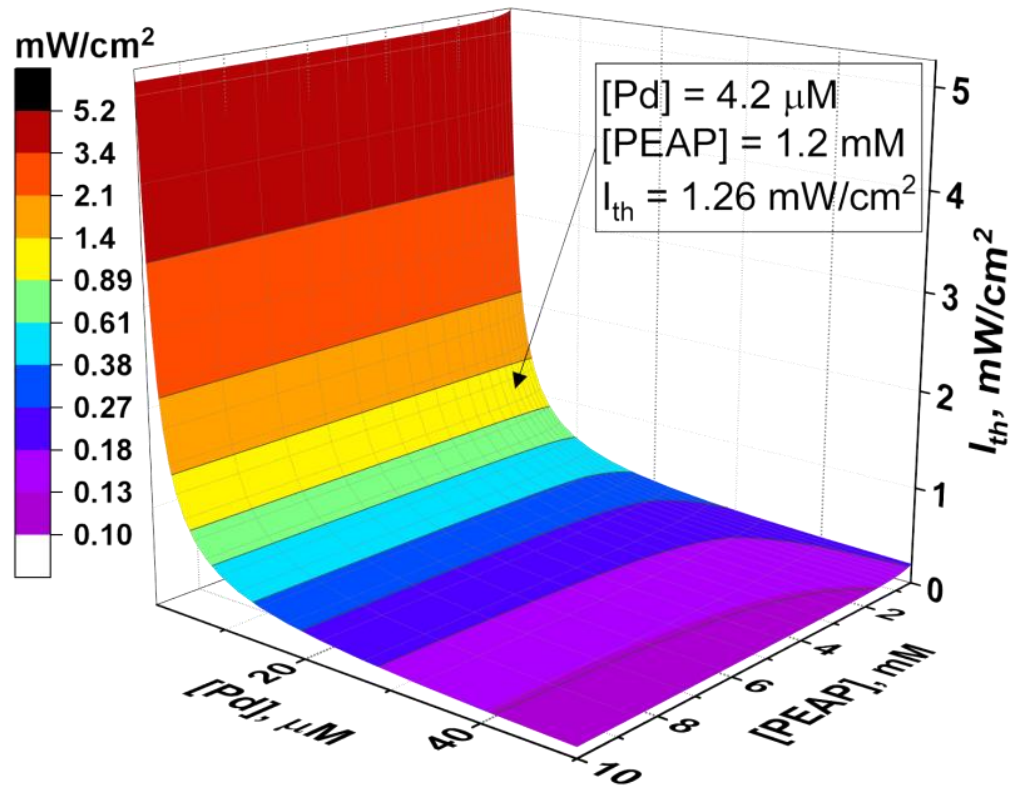

Figure S19. Kinetic rate model plot of PdTAPIP and PEAP in toluene (viscosity of $0.59 \mathrm{cP}$ ). 


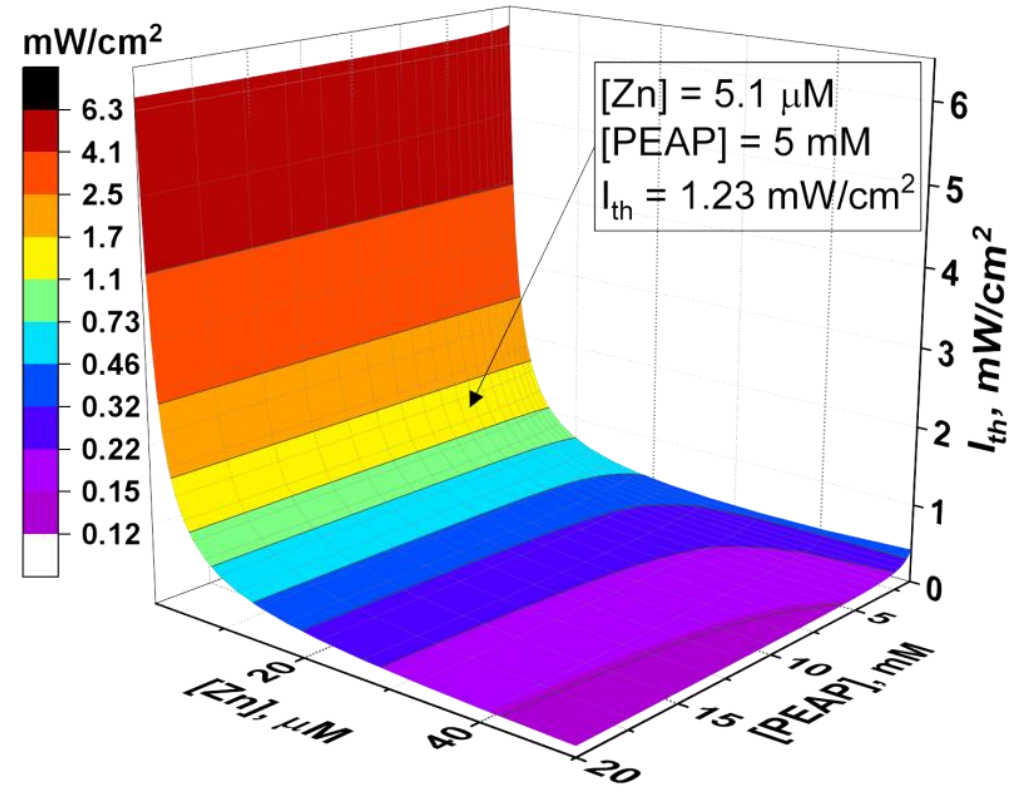

Figure S20. Kinetic rate model plot of ZnTAPIP and PEAP in toluene (viscosity of $0.59 \mathrm{cP}$ ). 


\section{References}

(1) Esipova, T. V; Rivera-Jacquez, H. J.; Weber, B.; Masunov, A. E.; Vinogradov, S. A. Stabilizing GStates in Centrosymmetric Tetrapyrroles: Two-Photon-Absorbing Porphyrins with Bright Phosphorescence. J. Phys. Chem. A 2017, 121 (33), 6243-6255. DOI: 10.1021/acs.jpca.7b04333.

(2) Fudickar, W.; Linker, T. Why Triple Bonds Protect Acenes from Oxidation and Decomposition. $J$. Am. Chem. Soc. 2012, 134 (36), 15071-15082. DOI: 10.1021/ja306056x.

(3) Olmsted, J. Calorimetric Determinations of Absolute Fluorescence Quantum Yields. J. Phys. Chem. 1979, 83 (20), 2581-2584. DOI: 10.1021/j100483a006.

(4) Crosby, G. A.; Demas, J. N. Measurement of Photoluminescence Quantum Yields. Review. J. Phys. Chem. 1971, 75 (8), 991-1024. DOI: 10.1021/j100678a001.

(5) Singh-Rachford, T. N.; Castellano, F. N. Photon Upconversion Based on Sensitized Triplet-Triplet Annihilation. Coord. Chem. Rev. 2010, 254 (21-22), 2560-2573. DOI: 10.1016/j.ccr.2010.01.003.

(6) Durandin, N. A.; Isokuortti, J.; Efimov, A.; Vuorimaa-Laukkanen, E.; Tkachenko, N. V; Laaksonen, T. Efficient Photon Upconversion at Remarkably Low Annihilator Concentrations in a Liquid Polymer Matrix: When Less Is More. Chem. Commun. 2018, 54 (99), 14029-14032. DOI: 10.1039/C8CC07592A.

(7) Durandin, N. A.; Isokuortti, J.; Efimov, A.; Vuorimaa-Laukkanen, E.; Tkachenko, N. V.; Laaksonen, T. Critical Sensitizer Quality Attributes for Efficient Triplet-Triplet Annihilation Upconversion with Low Power Density Thresholds. J. Phys. Chem. C 2019, 123 (37), 22865-22872. DOI: $10.1021 /$ acs.jpcc.9b08026.

(8) Gray, V.; Dreos, A.; Erhart, P.; Albinsson, B.; Moth-Poulsen, K.; Abrahamsson, M. Loss Channels in Triplet-Triplet Annihilation Photon Upconversion: Importance of Annihilator Singlet and Triplet Surface Shapes. Phys. Chem. Chem. Phys. 2017, 19 (17), 10931-10939. DOI: 10.1039/C7CP01368J. 